\title{
Acetylated nanocellulose for single-component bioinks and cell proliferation on 3D-printed scaffolds
}

\section{Ajdary, Rubina}

2019-07

Ajdary , R , Huan , S , Zanjanizadeh Ezazi , N , Xiang , W, Grande , R, Santos , H A \& Rojas , O J 2019 , ' Acetylated nanocellulose for single-component bioinks and cell proliferation on 3D-printed scaffolds ' , Biomacromolecules , vol. 20 , no. 7 , pp. 2770-2778 . https://doi.org/10.1021/a

http://hdl.handle.net/10138/304247

https://doi.org/10.1021/acs.biomac.9b00527

cc_by

publishedVersion

Downloaded from Helda, University of Helsinki institutional repository.

This is an electronic reprint of the original article.

This reprint may differ from the original in pagination and typographic detail.

Please cite the original version. 


\title{
Acetylated Nanocellulose for Single-Component Bioinks and Cell Proliferation on 3D-Printed Scaffolds
}

\author{
Rubina Ajdary, ${ }^{\dagger}$ Siqi Huan, ${ }^{\dagger}$ Nazanin Zanjanizadeh Ezazi, ${ }^{\ddagger}$ Wenchao Xiang, ${ }^{\dagger}$ Rafael Grande, ${ }^{\dagger}$

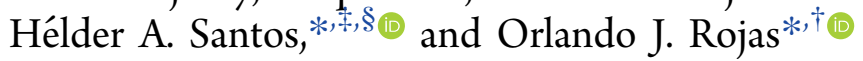

${ }^{\dagger}$ Department of Bioproducts and Biosystems, School of Chemical Engineering, Aalto University, P.O. Box 16300, FI 00076 Aalto,
Espoo, Finland

${ }^{\ddagger}$ Drug Research Program, Division of Pharmaceutical Chemistry and Technology, Faculty of Pharmacy and ${ }^{\S}$ Helsinki Institute of Life Science (HiLIFE), University of Helsinki, FI 00014 Helsinki, Finland

\section{Supporting Information}

ABSTRACT: Nanocellulose has been demonstrated as a suitable material for cell culturing, given its similarity to extracellular matrices. Taking advantage of the shear thinning behavior, nanocellulose suits three-dimensional (3D) printing into scaffolds that support cell attachment and proliferation. Here, we propose aqueous suspensions of acetylated nanocellulose of a low degree of substitution for direct ink writing (DIW). This benefits from the heterogeneous acetylation of precursor cellulosic fibers, which eases their

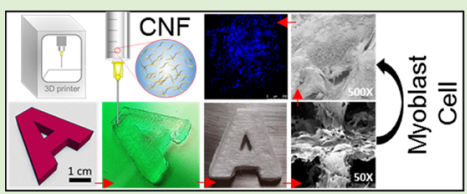
deconstruction and confers the characteristics required for extrusion in DIW. Accordingly, the morphology of related 3Dprinted architectures and their performance during drying and rewetting as well as interactions with living cells are compared with those produced from typical unmodified and TEMPO-oxidized nanocelluloses. We find that a significantly lower concentration of acetylated nanofibrils is needed to obtain bioinks of similar performance, affording more porous structures. Together with their high surface charge and axial aspect, acetylated nanocellulose produces dimensionally stable monolithic scaffolds that support drying and rewetting, required for packaging and sterilization. Considering their potential uses in cardiac devices, we discuss the interactions of the scaffolds with cardiac myoblast cells. Attachment, proliferation, and viability for 21 days are demonstrated. Overall, the performance of acetylated nanocellulose bioinks opens the possibility for reliable and scaleup fabrication of scaffolds appropriate for studies on cellular processes and for tissue engineering.

\section{INTRODUCTION}

The shear thinning behavior of nanocelluloses along with their excellent intrinsic mechanical strength and tailorable surface chemistry makes them promising for three-dimensional (3D) printing, particularly via extrusion-based direct ink writing (DIW $){ }^{1-8}$ Highly customizable structures are possible from DIW for applications in the biomedical, ${ }^{2,7-13}$ dental, ${ }^{13-17}$ packaging, ${ }^{18,19}$ foodstuff, ${ }^{20}$ construction, ${ }^{21}$ and aerospace fields. ${ }^{15,22-24}$ Although related technologies are still under development, nanocellulose-based bioprinting has clearly emerged for its potential in tissue engineering and regenerative medicine. $^{2}$ Recent efforts in such topics usually consider the use of nanocellulose in combination with other (bio)polymers, for example, in multicomponent ink formulations. ${ }^{2,3,25-28} \mathrm{On}$ the other hand, the formation of nanocomposite inks that exploit the nanocellulose networks to encapsulate nanoparticles and functional materials has also attracted recent attention. $^{29-31}$ For this purpose, post-treatments, such as crosslinking, are often applied, for example, to improve the mechanical integrity or to fulfill the requirements of the application..$^{3,32,33}$ For instance, the similarity and biocompatibility of nanocellulose scaffolds with the extracellular matrix (ECM) are essential for cell survival. These factors can be conveniently assessed by measuring cell viability and proliferation. ${ }^{34,35}$
Compared with other biopolymers, nanocelluloses are structurally similar to extracellular matrices. ${ }^{36}$ However, the major challenge in processing nanocelluloses is self-association and uncontrollable aggregation, which may be prevented by increasing the electrostatic charges or by surface functionalization. ${ }^{37}$ Unfortunately, most modifications make the 3D-printed materials susceptible to dimensional instability, for instance, upon drying or wetting. ${ }^{38}$ This is exacerbated if the inks are highly diluted, which is typical of nanocellulose suspensions, which form gels at low concentrations. ${ }^{39}$ These challenges also apply to compositions consisting of mixtures of nanocellulose with other biopolymers, such as alginate and heteropolysaccharides, which demand crosslinking after 3D printing to solidify the structure. ${ }^{1,40}$

TEMPO-oxidized nanocellulose has been reported as singlecomponent ink; however, double-crosslinking during and after $3 \mathrm{D}$ printing of the scaffolds is required. ${ }^{36}$ Likewise, aligned cellulose nanocrystals (CNC) were successfully 3D-printed although a reduced cell viability was observed. ${ }^{41}$ Although crosslinking or addition of complexing agents enhances the mechanical strength of the fabricated scaffolds, they come with significant drawbacks. Some of the most widely used

Received: April 17, 2019

Revised: May 20, 2019

Published: May 22, 2019 
Table 1. 3D Printing Parameters for CNF, TOCNF, and AceCNF

\begin{tabular}{lccc} 
ink & solid content, wt \% & needle diameter, mm & pneumatic pressure, $\mathrm{kPa}$ \\
CNF & 1.88 & 0.84 & 43 \\
TOCNF & 1.7 & 0.63 & 55 \\
AceCNF & 0.5 & 0.41 & 35 \\
\hline
\end{tabular}

crosslinkers in biomedicine, such as glutaraldehyde and genipin, are cytotoxic, and several washing steps are required to remove the unreacted groups that may affect the cell growth. Therefore, processing in the absence of crosslinking agents may be favored when a high stiffness is not required. ${ }^{42,43}$

The previous observations highlight standing and unresolved challenges relevant to ink formulations based on nanocelluloses, which otherwise would make them a preferred component for $3 \mathrm{D}$ printing, especially for the fabrication of monolithic structures. Hence, the aim of this study was to formulate single-component bioinks that did not require crosslinking to develop the strength or solidity of the printed structures. Here, we propose heterogeneous acetylation of wood fibers to ease their deconstruction into acetylated nanocellulose (AceCNF) for DIW. Dimensionally stable scaffolds were obtained by freeze-drying to facilitate sterilization and other processing steps.

In addition, we introduce AceCNF for the generation of 3Dprinted scaffolds for implantation in the human body. Being natural, easy to sterilize, ${ }^{44}$ and given their high stability, porosity, ${ }^{45}$ and hemocompatibility (or blood compatibility), ${ }^{46,47}$ nanocelluloses present unique opportunities as biomaterials in 3D scaffold applications. Bacterial nanocellulose was previously introduced as an option for cardiac patches, showing favorable elasticity and a negligible inflammatory reaction. ${ }^{48,49}$ Also, magnetically aligned CNC scaffolds successfully encapsulated skeletal muscle myoblast cells to form highly oriented myotubes. ${ }^{50}$ The interaction of several types of cells has been reported with nanocellulosebased composites, from stem cells and fibroblast cells to skeletal myoblasts. ${ }^{51-53}$ However, an issue that still remains for elucidation is about how 3D plant-based nanocellulose scaffolds interact with cardiac myoblast cells. Here, we determined the microstructure, the cardiac myoblast viability, proliferation, and attachment on AceCNF to examine the potential for cardiac tissue engineering, and the results were compared with those obtained from unmodified (CNF) and TEMPO-oxidized (TOCNF) nanocelluloses.

\section{EXPERIMENTAL SECTION}

Materials. Cellulose nanofibrils (CNF) were produced through disintegration of never-dried, fully bleached, and fines-free birch wood fibers, as reported elsewhere. ${ }^{54}$ Never-dried Kraft fibers obtained from birch wood were utilized to produce AceCNF following our previously reported method through partial heterogeneous acetylation. ${ }^{55}$ The acetylation degree of substitution (DS) of AceCNF was 0.6. TOCNF was prepared from never-dried birch fibers by TEMPOmediated oxidation (2,2,6,6-tetramethylpiperidine-1-oxyl). Cellulose fibers were first suspended in water at $17.03 \mathrm{wt} \%$, and then TEMPO $(0.013 \mathrm{mmol} / \mathrm{g})$ and sodium bromide $(0.13 \mathrm{mmol} / \mathrm{g})$ were added. Sodium hypochlorite $(\mathrm{NaClO})(5 \mathrm{mmol} / \mathrm{g})$ was gently added to the fiber suspension and the $\mathrm{pH}$ was adjusted to 10 by adding $0.1 \mathrm{M}$ $\mathrm{NaOH}$. The mixture was stirred for $6 \mathrm{~h}$ at room temperature. The resulting TEMPO-oxidized fibers were thoroughly washed with deionized water up to neutral $\mathrm{pH}$. Their fibrillation was carried out with a microfluidizer (M-110P, Microfluidics In., Newton, MA) using four passes at a pressure of 1500 bars. The viscous and translucent hydrogel was then concentrated to $1.7 \mathrm{wt} \%$ by evaporating water under stirring at room temperature. The carboxylic group content on the surface of the obtained TOCNF was $1.4 \mathrm{mmol} / \mathrm{g}$, as determined by conductometric titration following previous reports. ${ }^{56,57}$ Sodium chloride $(\geq 99 \%)$ was purchased from Sigma-Aldrich. Milli-Q water was purified with a Millipore Synergy UV unit $(18.2 \mathrm{M} \Omega \mathrm{cm})$ and used throughout the experiments. Other solvents include ethanol (ETAX Aa 99.5\%) and acetone (AnalaR NORMAPUR 99.8\%). Fetal bovine serum (FBS), Dulbecco's modified Eagle medium (DMEM), L-glutamine, nonessential amino acids (NEAA), and penicillinstreptomycin were purchased from HyClone.

Nanocellulose Properties. Morphology. Atomic force microscopy (AFM, MultiMode 8 Scanning Probe Microscope, Bruker AXS Inc.) was used to analyze the topological features of the different nanofibrils (unmodified CNF, TOCNF, and AceCNF). The given nanofibril suspension $(0.001 \mathrm{wt} \%)$ was spin-coated on polyethyleneimine-coated mica. The nanofibril-coated substrates were dried overnight at room temperature before imaging. The AFM scans $(1 \times$ $\left.1 \mu \mathrm{m}^{2}\right)$ were collected under tapping mode in air with silicon cantilevers (NSC15/AIBS, MicroMasch). Three different spots of each sample were imaged; no image processing was adopted except flattening.

Surface Charge. To evaluate the surface charge, suspensions of nanocelluloses at a concentration of $0.1 \mathrm{wt} \%$ in $5 \times 10^{-3} \mathrm{M}$ sodium chloride were used to measure the $\zeta$-potential by a dip cell on a Malvern, Zetasizer ZS.

Rheological Behavior. The viscosities of the inks were studied with a dynamic rotational rheometer (MCR 302, Anton Paar, Germany), using parallel plates (PP25) with the gap fixed at $1 \mathrm{~mm}$. The change of viscosity was monitored by increasing the shear rate from $10^{-2}$ to $10^{2} \mathrm{~s}^{-1}$. For dynamic viscoelastic quantification, the linear viscoelastic range was measured with a strain sweep ranging from $10^{-2}$ to $10^{2} \%$ at a fixed frequency of $10 \mathrm{rad} / \mathrm{s}$. The same rheometer was used to conduct frequency sweep $\left(10^{-1}\right.$ to $\left.10^{2} \mathrm{rad} / \mathrm{s}\right)$ tests with a fixed gap of $0.5 \mathrm{~mm}$ and at a constant strain of $0.5 \%$, which is in the range of the linear viscoelastic region. The data obtained from the dynamic mechanical spectra and the storage $\left(G^{\prime}\right)$ and loss $\left(G^{\prime \prime}\right)$ moduli were plotted as a function of frequency. RheoPlus was used for data processing. All measurements were conducted at $23{ }^{\circ} \mathrm{C}$.

3D Printing of Nanocellulose Inks. The single-component nanocellulose inks, namely, CNF (0.5, 1.7, and 1.88 wt \%), TOCNF $(0.5,1.7$, and $2.1 \mathrm{wt} \%)$, and AceCNF (0.5 wt \%), were screened according to the rheology data. The optimal concentrations of CNF, TOCNF, and AceCNF inks in the experiments were 1.88, 1.7, and 0.5 wt $\%$, respectively. Later, the similar apparent viscosity profiles at these concentrations will be discussed.

A BIO X Bioprinter (CELLINK, Gothenburg, Sweden) equipped with a pneumatic print head was used to extrude single filaments and to form the 3D structures. All printed samples had a rectilinear infill pattern and $25 \%$ infill density. The system utilized the clear pneumatic $3 \mathrm{~mL}$ syringe provided and sterile blunt needles $19 \mathrm{G}, 20 \mathrm{G}$, and $25 \mathrm{G}$ from CELLINK. The sizes of the nozzle tips were 0.41, 0.63, and 0.84 $\mathrm{mm}$, respectively. The solid support used for 3D printing consisted of plastic Petri dishes (100 mm diameter).

Printing parameters including the nozzle size, print head speed, and extrusion pressure (Table 1) were adjusted to achieve suitable conditions for 3D printing of nanocellulose inks, according to the quality and fidelity of the 3D-printed structure. The ink composition (concentrations of components) and formulation (nature of the component and other factors) were the variables considered along with appropriate processing conditions that were selected according to the rheology observed for the inks. However, it is likely that several combinations (composition, formulation, and processing parameters) 

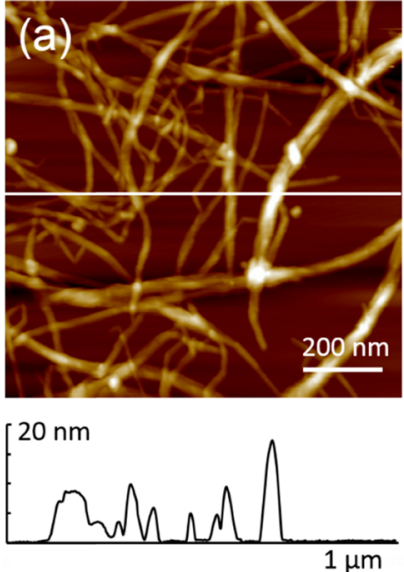
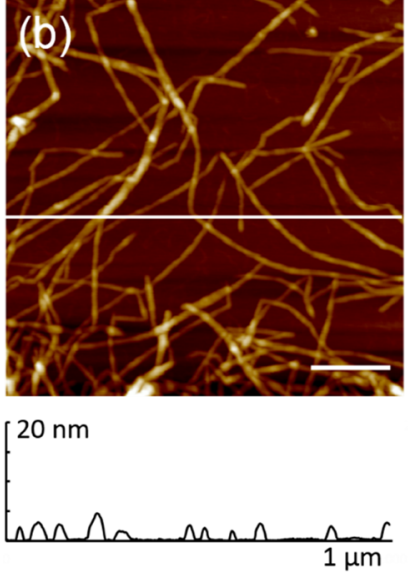

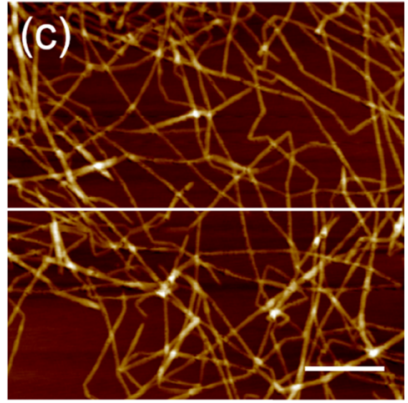

$20 \mathrm{~nm}$

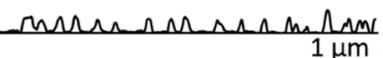

Figure 1. Atomic force microscopy height images of (a) CNF, (b) TOCNF, and (c) AceCNF. The scale bar is $200 \mathrm{~nm}$.

might lead to better 3D-printed structures. After 3D printing, the samples were frozen overnight at $-18{ }^{\circ} \mathrm{C}$ followed by vacuum drying for $48 \mathrm{~h}$ at $-49{ }^{\circ} \mathrm{C}$.

Characterization of 3D-Printed Objects. Microstructure. The microstructures of extruded filaments and scaffolds after freeze-drying were observed by scanning electron microscopy (SEM, Zeiss Sigma VP, German) operated under vacuum and at an accelerated voltage of $2 \mathrm{kV}$. The dry samples were fixed on metal stubs using a carbon tape and coated with a $4 \mathrm{~nm}$ layer of gold palladium alloy using a LECIA EM ACE600 sputter coater.

Shrinking and Swelling of the 3D-Printed Scaffolds. For a comparison, all of the scaffolds were 3D-printed with a nozzle diameter of $0.84 \mathrm{~mm}$ to attain equal geometry including the number of layers, layer thickness, and infill density. The choice of this nozzle diameter supported the least precise ink, CNF (Table 1). The visual appearance of the samples was recorded and measured with a ruler before and after drying. The extent of structure shrinkage was calculated after drying at room temperature or upon sublimation. The swelling capacities of the 3D-printed scaffolds obtained with the three types of nanocelluloses were measured using the tea-bag method. Freeze-dried scaffolds were placed inside a tea bag and immersed in excess water for $24 \mathrm{~h}$, following which they were weighted after 10 $\mathrm{min}$ of drainage. The same procedure was followed for three blank tea bags to obtain the absorption capacity per gram of tea bag. The water absorbed by the blank was subtracted from the total water absorption of the scaffold to obtain the effective water sorption capacity

$$
\text { swelling capacity }=\frac{w_{\mathrm{stw}}-w_{\mathrm{sd}}-w_{\mathrm{td}} w_{\mathrm{wgt}}}{w_{\mathrm{sd}}}
$$

where $w_{\text {stw }}$ is the weight of the wet sample and the tea bag, $w_{\text {sd }}$ is the weight of the dry sample, $w_{\mathrm{td}}$ is the weight of the dry tea bag, and $w_{\mathrm{wgt}}$ is the water absorption per gram of tea bag ( $g$ water/g tea bag). Three replicates were carried out and the average values were reported.

Cell Viability and Proliferation of the 3D-Printed Scaffolds. The viability and proliferation of cardiomyoblast H9C2 (American Type Culture Collection CRL-1446) on the surface of the 3D-printed nanocellulose scaffolds were accessed for 21 days, using the AlamarBlue Cell Viability Reagent (Thermo Fisher Scientific), which is based on the reduction of resazurin to fluorescent resorufin. The selection of this type of cells was made considering the potential for the tested nanocelluloses in cardiac biomedical devices. The samples were sterilized with UV irradiation for $3 \mathrm{~h}$ and then immersed in the cell culture medium, DMEM $+10 \%$ FBS, $1 \%(\mathrm{w} / \mathrm{v}) \mathrm{L}-$ glutamine, $1 \%(\mathrm{w} / \mathrm{v})$ NEAA, and penicillin-streptomycin (100 IU/ $\mathrm{mL}$ ), to mimic the biological environment. About $10000 \mathrm{H} 9 \mathrm{C} 2$ cells were seeded on top of each type of scaffold fabricated with CNF, TOCNF, and AceCNF. Light cellulose samples were trapped by Pyrex cylinders (Thermo Fisher Scientific) at the bottom of each well. Empty wells of positive controls (only cells with Pyrex cylinders) and negative controls (containing 1\% Triton X-100) were incubated at 37
${ }^{\circ} \mathrm{C}$ in $5 \% \mathrm{CO}_{2}$ in 48 -well plates (five replicates). At each time point, the DMEM was discarded and 10\% AlamarBlue-DMEM was added to each well and incubated for $6 \mathrm{~h}$ in the dark. Resorufin was extracted and inserted into new 96-well plates, and the cell viability and proliferation were measured by a Varioskan Flash plate reader (ThermoFisher). The calculations were based on comparisons with the positive and negative control wells. ${ }^{58}$ Additionally, 20000 myoblasts were seeded on nanocellulose samples for imaging the cell population in DMEM $+10 \%$ FBS by a fluorescence optical microscope. Samples were washed on the fourth day with phosphate buffered saline (PBS) three times and then fixed with $4 \%$ paraformaldehyde (PFA, Sigma-Aldrich), for $15 \mathrm{~min}$ at $37{ }^{\circ} \mathrm{C}$. Thereafter, $2.48 \mu \mathrm{g} / \mathrm{mL}$ of the fluorescence stain $4^{\prime}, 6$-diamidino-2phenylindole (DAPI) (Thermo Fisher) was added to label the DNA for $3 \mathrm{~min}$. Samples were washed and mounted with the Vectashield antifade mounting medium (Vector Laboratories) on an 8-well chambered cover glass. The samples were imaged by a Leica DM5000 fluorescence optical microscope (Leica Microsystems, Germany), with an objective of $10 \times$.

Cell Attachment and Morphology on the 3D-Printed Scaffolds. Cardiomyoblast attachment and morphology on the nanocellulose samples were studied in vitro during 4 days by SEM. Briefly, samples were sterilized under UV light for $3 \mathrm{~h}$ and then immersed in DMEM + 10\% FBS, with 1\% (w/v) L-glutamine, 1\% (w/ v) NEAA, and penicillin-streptomycin $(100 \mathrm{IU} / \mathrm{mL})$, for $24 \mathrm{~h}$ when 20000 cells were counted and seeded on each type of sample in 48well plates. Samples were trapped on the bottom of each well by Pyrex cylinders. On day 4, samples were washed with PBS buffer three times and then fixed with $2.5 \%$ glutaraldehyde in PBS at $37^{\circ} \mathrm{C}$ for $30 \mathrm{~min}$. Excessive glutaraldehyde was washed away with PBS two times. Postfixation and dehydration of cells were performed using $1 \%$ osmium tetroxide in PBS for $1 \mathrm{~h}$ and 50, 70, 96, and $100 \%$ of ethanol, respectively. Then, they were dried by the critical-point drying method and coated with $4 \mathrm{~nm}$ of gold palladium alloy using a LECIA EM ACE600 sputter coater. The samples were imaged by SEM (Zeiss Sigma VP, Germany) under vacuum and at an accelerated voltage of 2 $\mathrm{kV}$.

\section{RESULTS AND DISCUSSION}

Single-Component Nanocelluloses for DIW. Nanofibrils (CNF, TOCNF, and AceCNF) were well-dispersed, with no apparent fiber bundling (Figure 1). The negative surface charges (measured by the $\zeta$-potential) corresponded to $-47,-82.5$, and $-73.5 \mathrm{mV}$, respectively. Similar values were reported for unmodified CNF and TOCNF. ${ }^{59,60}$ The presence of surface charges reduced aggregation, easing the processing and favoring the retention of the structure after extrusion. In addition, the large axial aspect of the nanofibers contributes to mechanical entanglement. ${ }^{37}$ The atomic force microscopy 

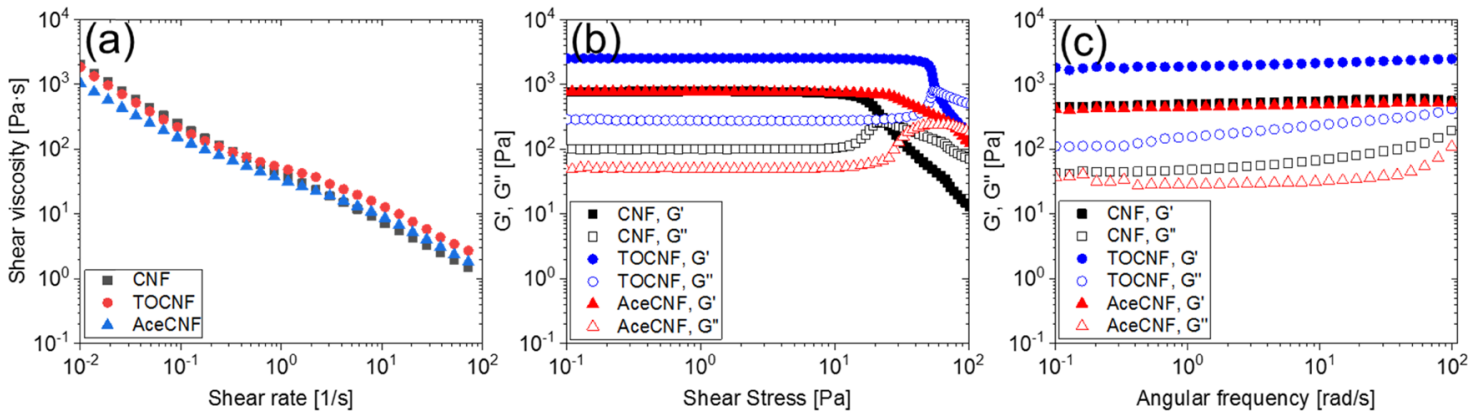

Figure 2. (a) Flow curves for the apparent shear viscosity as a function of the shear rate. (b) Oscillatory rheological behavior of nanocellulose inks. (c) Moduli (storage modulus $G^{\prime}$ and loss modulus $G^{\prime \prime}$ ) of $1.88 \mathrm{wt} \% \mathrm{CNF}, 1.7 \mathrm{wt} \%$ TOCNF, and $0.5 \mathrm{wt} \%$ AceCNF. All of the measurements were performed at constant temperature $\left(23{ }^{\circ} \mathrm{C}\right)$.
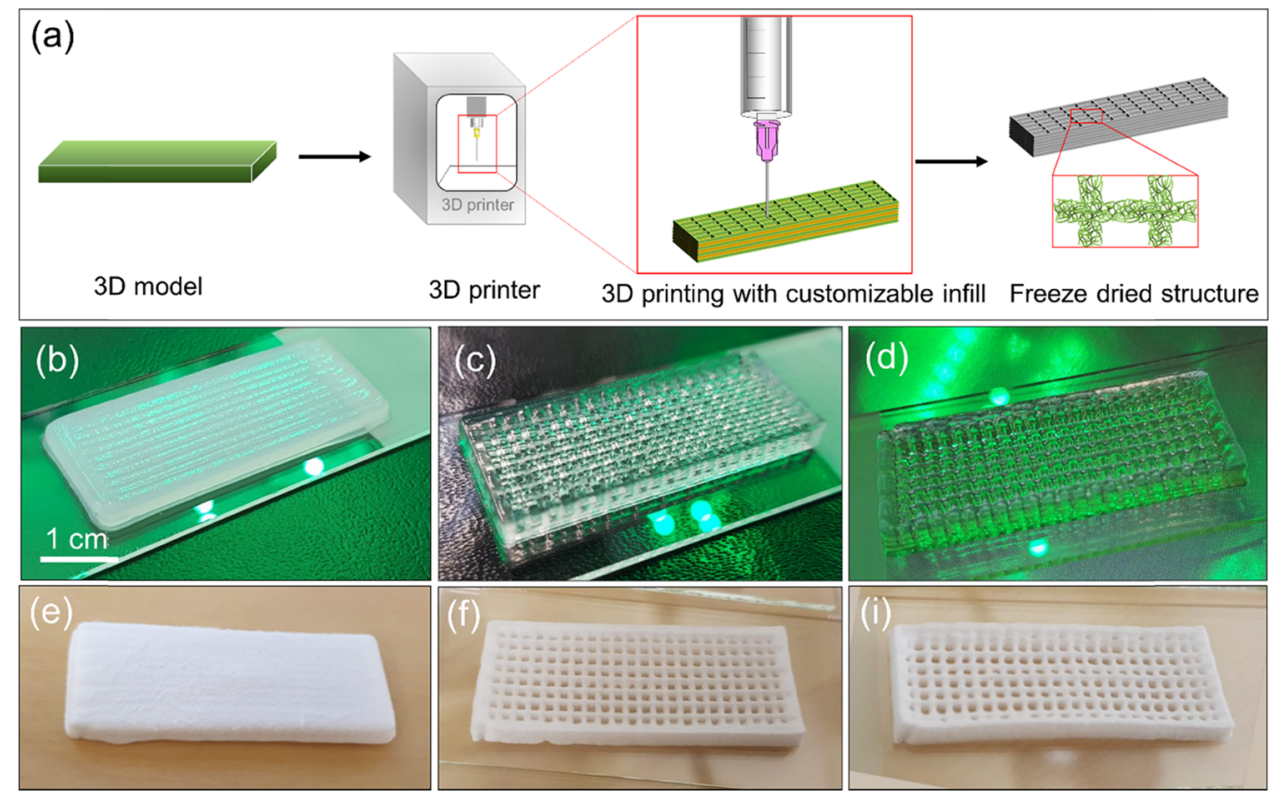

Figure 3. (a) Schematic illustration of DIW using nanocelluloses. A model is 3D-printed in layers with defined infill in a grid lattice structure and freeze-dried to retain the structure. The fidelity of the 3D-printed structures is shown in (b) CNF, (c) TOCNF, and (d) AceCNF, before drying. Such architectures are shown after drying in (e), (f), and (i) for the respective bioinks.

images of three nanocellulose samples and the amplitude error in $5 \times 5 \mu \mathrm{m}^{2}$ is included in Figure $\mathrm{S} 1$ of the Supporting information.

For a comparison, the rheological behavior of aqueous suspensions of nanocelluloses at given concentrations were assessed (Figure S2, Supporting information). The solid contents of CNF, TOCNF, and AceCNF were selected to yield similar flow profiles, as shown in Figure 2a, namely, inks with similar viscosity behaviors for 3D printing were obtained. The lowest solid content, 0.5 wt $\%$, corresponded to the AceCNF hydrogel, and a remarkable viscosifying effect was shown with increased concentration, to the point of preventing ink extrusion. The concentrations of CNF and TOCNF were adjusted accordingly to exhibit similar apparent viscosities. All nanocellulose inks underwent shear-thinning; values of the apparent viscosity in the range of shear rates between $10^{-2}$ and $10^{2} \mathrm{~s}^{-1}$ were noted as appropriate for direct ink writing. ${ }^{61-63}$ Oscillatory rheology was conducted to study the dynamic mechanical behavior of the nanocellulose inks. The effect of stress amplitude on the nanocellulose ink is illustrated in Figure 2 b. The $G^{\prime}>G^{\prime \prime}$ of all samples indicates the gel-like behavior of all nanocellulose inks under a wide range of shear stresses. The sharp decrease of $G^{\prime}$ beyond the linear viscoelastic region is due to structural breakdown of the nanocelluloses under extensive deformation. ${ }^{61}$ The critical shear stress values at which the ink network displayed a nonlinear viscoelastic behavior corresponded to 11, 43, and 17 $\mathrm{Pa}$ for CNF, TOCNF, and AceCNF, respectively. The frequency sweep tests were performed at $10 \mathrm{~Pa}$ to investigate the stability of the inks along the entire frequency range from $10^{-1}$ to $10^{2} \mathrm{rad} / \mathrm{s}$. According to Figure $2 \mathrm{c}$, all inks showed a dominant elastic behavior with $G^{\prime}>G^{\prime \prime}$, by about 1 order of magnitude. Both $G^{\prime}$ and $G^{\prime \prime}$ displayed a large viscoelastic plateau along the entire frequency range, taken as an indication of stable inks. The absence of a cross-point between $G^{\prime}$ and $G^{\prime \prime}$ confirms the stability of the nanocellulose inks. Furthermore, the frequency-independent elastic modulus profile points toward the solid-like behavior of the samples.

DIW and 3D-Printed Structures. A schematic illustration of the DIW process with nanocelluloses is shown in Figure 3 a. A $3 \mathrm{D}$ model was first imported to the $3 \mathrm{D}$ printer, and the parameters were adjusted according to the ink, including printing speed, extrusion pressure, structure infill, and nozzle diameter. Each nanocellulose ink was applied at the respective 


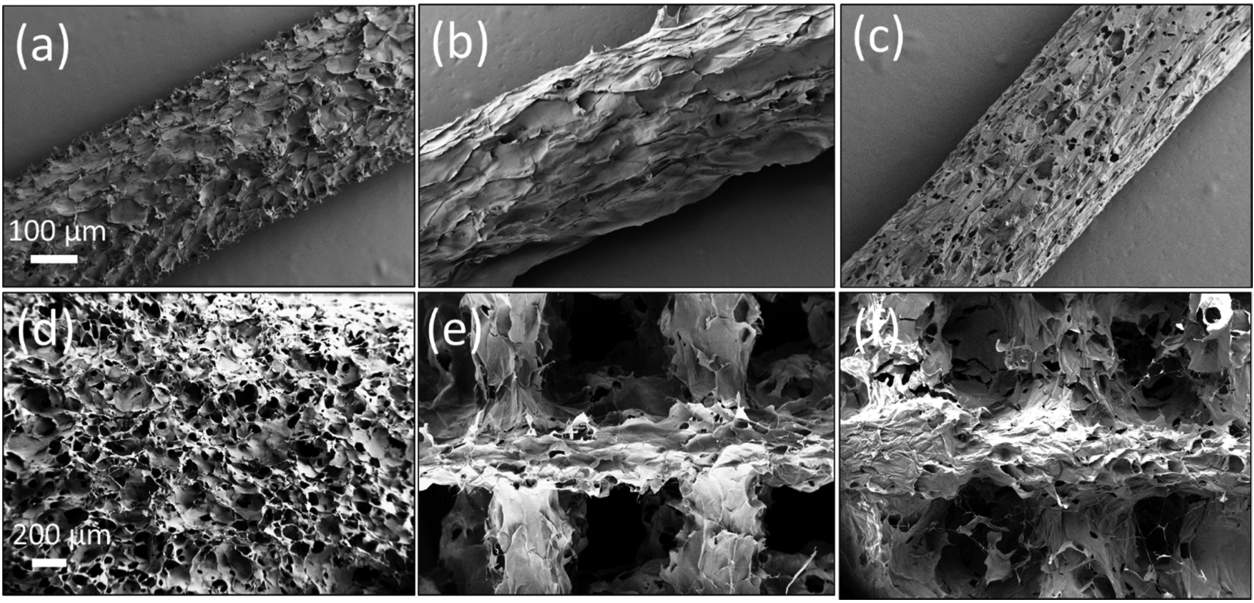

Figure 4. Microstructures of extruded (a) CNF, (b) TOCNF, and (c) AceCNF filaments. (d) 3D-printed CNF scaffold showing the swelled filament merging upon extrusion. The scaffolds corresponding to (e) 3D-printed TOCNF and (f) AceCNF are also shown. The scale bars for (ac) and (d-f) are 100 and $200 \mu \mathrm{m}$, respectively.

solid content, with no addition of any other component. As shown in Figure $3 b-i$, the rectangular open spaces within the grid structure produced in the CNF scaffold were deformed at least partially and, in some cases, collapsed after 3D printing and freeze-drying. In contrast, the structures produced with AceCNF and TOCNF maintained the structure under both wet and dry conditions, indicating that the high fidelity of 3Dprinted structures were retained for these bioinks. They also maintained the structure after extrusion, resulting in the formation of 3D-printed shapes that closely followed the details of the design. TOCNF and AceCNF were visually stable after 3D printing of the grid lattice and honeycomb infill patterns; layer deposition was up to a height of $2 \mathrm{~cm}$ in these samples (Figure S3, Supporting information).

The carboxyl groups in TOCNF improve the dispersion and stability of nanocellulose suspensions, and the relatively higher axial aspect of the fibrils is also beneficial for achieving better nanofibril entanglement. ${ }^{64}$ Accordingly, it can be reasonably hypothesized that a similar effect applies to AceCNF. From Figure 1, it is observed that AceCNF nanofibrils are thinner, which facilitates a more significant entanglement. Most importantly, the concentration of AceCNF was three times lower than that of TOCNF. Thus, both the surface charge and the axial aspect contribute to better retention of the structure upon extrusion. ${ }^{65}$

Microstructures of 3D-Printed Scaffolds. The microstructure of the extruded filaments and 3D-printed scaffolds are displayed in Figure $4 a-c$. Single filaments clearly display different surface roughnesses and porosities. The CNF ink produced filaments with rough surfaces, whereas those of AceCNF ink were highly porous but with a lower surface roughness. TOCNF filaments were also rough and showed a tendency to deform (flatten) on the support after 3D printing (Figure S4, Supporting information). The microstructures of the scaffolds are shown in Figure $4 \mathrm{~d}-\mathrm{f}$. Although the freezedried TOCNF and AceCNF scaffolds clearly displayed the different printed layers, no apparent layer separation was observed in the SEM images. The 3D-printed scaffolds with CNF showed clear signs of layer fusion after printing as a result of extensive swelling of the extruded ink.

Shrinking and Swelling Behavior. Regulation of cellular activities in ECMs is closely associated with water retention stability. $^{34,35}$ To investigate related effects, all three inks were extruded with the same needle $(0.84 \mathrm{~mm}$ diameter $)$ to ensure an equal number of layers for each structure printed from the respective nanocellulose. Thereafter, the degree of shrinkage and water swelling of the $3 \mathrm{D}$-printed scaffolds $(20 \times 20 \times 3$ $\mathrm{mm}^{3}$, infill density $25 \%$ ) were determined. The 3D-printed scaffolds were freeze-dried or dried at ambient temperature (ca. 1 week was needed for drying) before swelling tests. As shown in Figure 5, the samples dried at room temperature

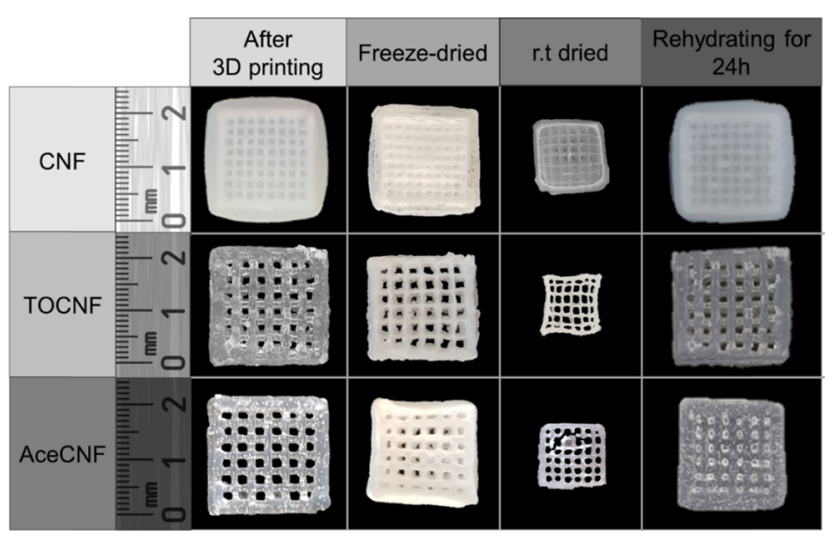

Figure 5. CNF, TOCNF, and AceCNF scaffolds in the wet state soon after printing, after freeze-drying, after room temperature drying (24 $\mathrm{h}$ ), and after rehydration ( $24 \mathrm{~h}$ of immersion in water) of the freezedried samples.

underwent a $45-50 \%$ shrinkage, compared with $0-15 \%$ for the samples that were freeze-dried. The latter method retained more effectively the shape of the printed structure. The structures printed with AceCNF deswelled extensively upon ambient drying, given the fact that the precursor nanomaterial was diluted to the largest degree. However, the free-dried samples retained accurately the initial $3 \mathrm{D}$ geometry.

The swelling capacities of the 3D-printed scaffolds were determined by comparing the weight after $24 \mathrm{~h}$ of drying at room temperature and rehydration. TOCNF scaffolds displayed the largest swelling capacity, $14 \pm 0.2 \mathrm{~g} / \mathrm{g}$, as explained by the presence of carboxyl groups. ${ }^{65,66}$ In contrast, the less hydrophilic nanocellulose, AceCNF, displayed the lowest swelling degree, $5 \pm 0.3 \mathrm{~g} / \mathrm{g}$ (the unmodified CNF absorbed around $11 \pm 0.7 \mathrm{~g} / \mathrm{g}$ ). Remarkably, all three bioinks 
(a)

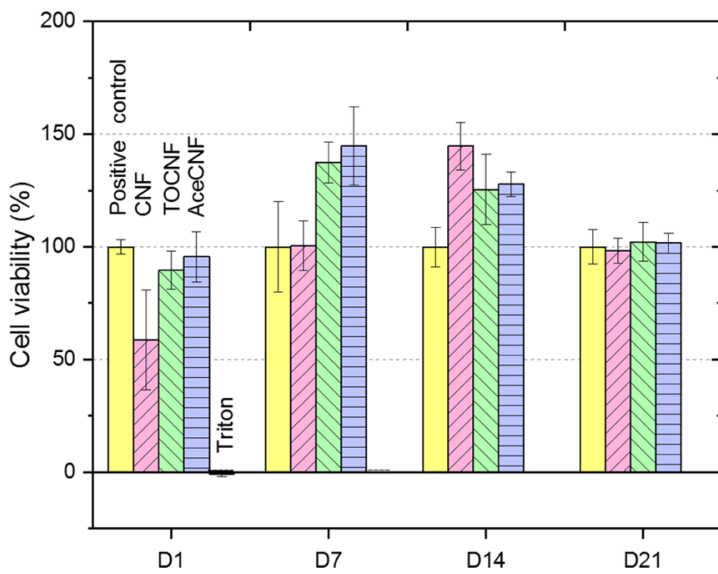

(b)

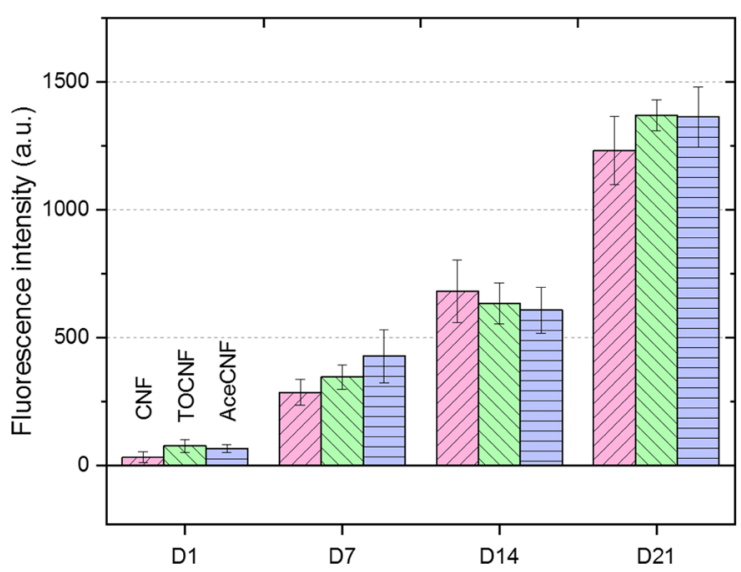

Figure 6. (a) H9C2 viability of nanocellulose samples during 21 days in DMEM + FBS 10\%, showing high biocompatibility and proliferation by an AlamarBlue assay. Note the positive control consisting of pure myoblast and Pyrex cylinders in DMEM $+10 \%$ FBS without the nanocellulose samples. (b) H9C2 proliferation of nanocellulose samples inside DMEM + FBS 10\% for 21 days based on the fluorescence intensity obtained from the AlamarBlue assay.
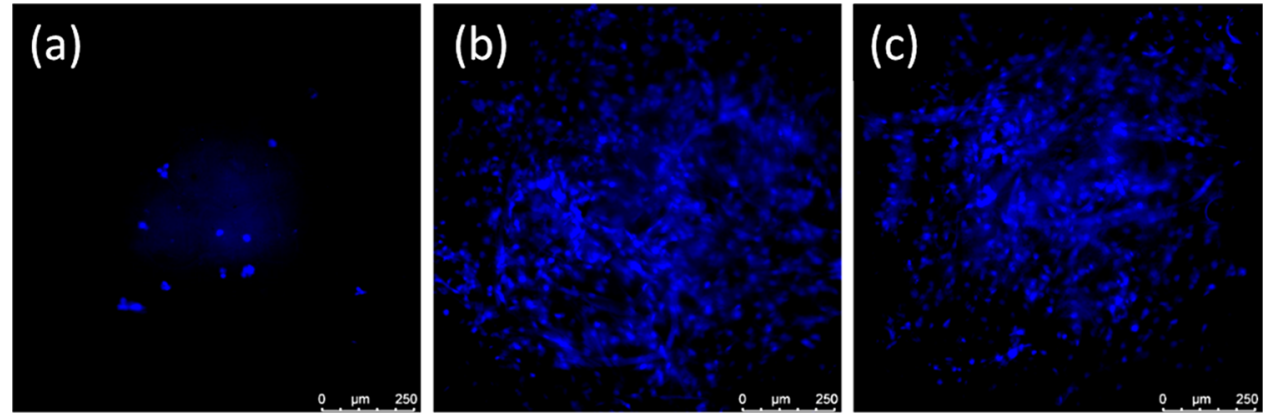

Figure 7. Fluorescence microscopy images of the cell populations seeded on nanocellulose samples in DMEM $+10 \%$ FBS on day 4: (a) CNF, (b) TOCNF, and (c) AceCNF. Cells were fixed with 4\% PFA, and the nuclei of cells were stained with DAPI. The comparison shows a high population of $\mathrm{H} 9 \mathrm{C} 2$ cells on the AceCNF 3D structure, almost as much as that on TOCNF, whereas a lower population is observed on CNF.

retained their structure and did not disintegrate even after $24 \mathrm{~h}$ of immersion in water. For a given geometry, and compared with AceCNF scaffolds, those produced from CNF and TOCNF have a more than three times higher solid content. Therefore, on this basis, it was expected that CNF and TOCNF would retain their shape better upon rehydration. The observations indicate that single-component nanocellulose inks produce scaffolds that recover their shape after rehydration of the freeze-dried samples, which is of interest for subsequent operations such as sterilization, transport, and final deployment, for example, for cell culturing. This latter aspect is discussed next.

Cell Viability and Proliferation. Nanocelluloses have shown great promise in biomedical applications, given their biocompatibility and low toxicity. ${ }^{67}$ Myoblast cells seeded on $\mathrm{CNF}$, TOCNF, and AceCNF scaffolds were assessed by AlamarBlue assay for 21 days to monitor the cell viability and proliferation. Resorufin extracted from each sample was measured against the positive control. As shown in Figure $6 a$, the samples showed biocompatibility for 21 days, with extensive cell proliferation (Figure $6 \mathrm{~b}$ ). The highly porous, interconnected structures of each scaffold type can improve the cell penetration in the structure and assist in nutrient transport to the cells as well as in the transport of metabolic waste. ${ }^{27,68}$ Although the results indicate a similar behavior for all nanocellulose scaffolds, as far as the biocompatibility and induced proliferation of the cells on the surface are concerned, TOCNF and AceCNF produced higher cell viability compared with the positive controls and CNF for days 1 and 7 . Qualitative results indicate a lower cell population on CNF in the fluorescence microscope images taken on day 4 (Figure $7 a-c)$, supporting the cell viability results, which demonstrate the lower capability of CNF for hosting the cells compared with the other two cellulose types in less than 7 days. The CNF ink creates an integrated 3D scaffold with no void spaces within the grid lattice structure (Figure 4d). Moreover, the lower surface charge of CNF may result in a lower degree of attachment and viability of the cells in the early stages. ${ }^{69}$ However, at days 14 and 21, all three 3D-printed nanocellulose-based samples showed about two and four times more extensive cell proliferation compared with that in the first week. This effect is hypothesized to be a result of the negative surface charges ${ }^{69,70}$ and the hydrophilicity of the samples. ${ }^{71}$ Martins et al. showed that the $\mathrm{C} 2 \mathrm{C} 12$ mouse muscle myoblast proliferation was improved on the negatively charged surfaces of poly(vinylidene fluoride) fibers. Other authors have indicated an improvement of fibroblast cell adhesion for TEMPO-oxidized nanocellulose. ${ }^{27}$

Cell Morphology and Attachment. Cell morphology and attachment of cardiomyoblasts were examined during 4 days in DMEM + 10\% FBS by SEM (Figure 8). Figure 8a displays the CNF control at $500 \times$ magnification and Figure $8 \mathrm{~d}$ 


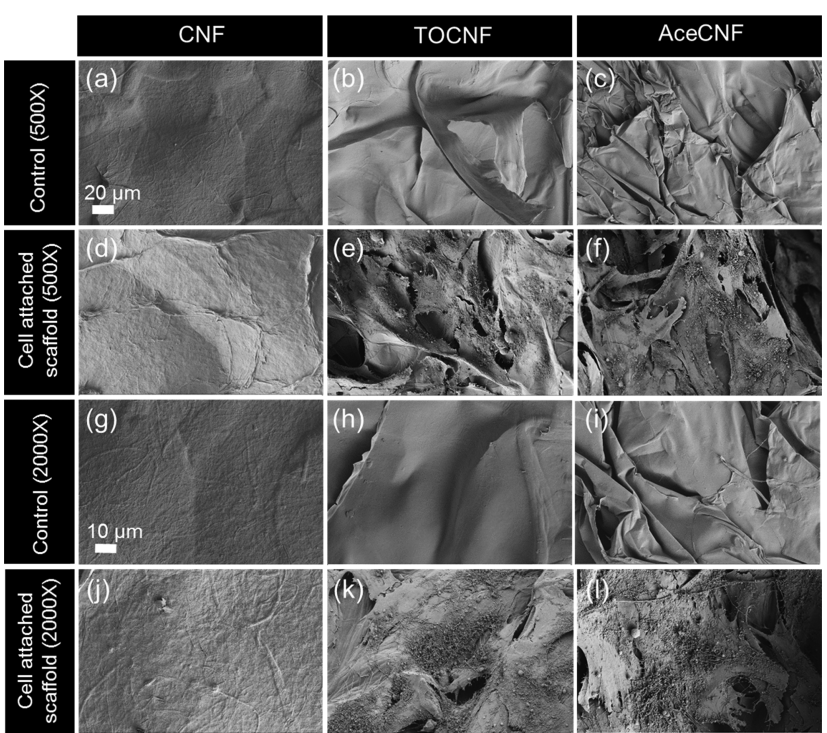

Figure 8. Cardiomyocyte attachment and morphology on CNF (a, d, g, j), TOCNF (b, e, h, k), and AceCNF (c, f, i, l). (a, b, c, g, h, i) Control samples without cells and (d, e, f, j, k, l) nanocellulose samples with attached cells on the surface. The magnification in $(a-f)$ is 500 and that in $(\mathrm{g}-1)$ is $2000 \times$.

shows the scaffold under cell attachment conditions at a similar magnification. Figure $8 \mathrm{~g}-\mathrm{j}$ show $\mathrm{CNF}$ control and cellattached samples at a higher magnification of $2000 \times$. The CNF 3D structure was not able to host the cells during 4 days. The same pattern was observed in the fluorescence imaging (Figure 7a). The results in Figure $7 \mathrm{~b}$ and $\mathrm{c}$ indicate cell interaction and adherence on TOCNF and AceCNF. Figure $8 \mathrm{~b}-\mathrm{h}$ show the control samples at $500 \times$ and $2000 \times$ magnifications and Figure $8 \mathrm{e}-\mathrm{k}$ show the cell-attached scaffolds at the mentioned magnifications. SEM images of the AceCNF control and cell-attached samples are displayed in Figure $8 \mathrm{c}, \mathrm{f}(500 \times)$ and Figure $8 \mathrm{i}, 1 \mathrm{(2000 \times )}$. As discussed above, compared with $\mathrm{CNF}$, the higher surface charges and surface areas of TOCNF and AceCNF provide more suitable matrices for cell infiltration and attachment. These results correlate well with the cell viability results, showing cell attachment on the 3D structures in less than 7 days of cell seeding.

\section{CONCLUSIONS}

The 3D-printed, single-component scaffolds were produced from acetylated nanocellulose obtained by heterogeneous acetylation of wood fibers. Scaffolds were freeze-dried to facilitate sterilization and cell seeding in further steps. The scaffolds were highly stable and did not require further crosslinking steps or the addition of other compounds. The morphology, rheological behavior, and microstructure of the acetylated nanocellulose were compared with those of unmodified and TEMPO-oxidized nanocelluloses. Cell viability and proliferation tests were performed for 21 days to investigate the interaction of cells with the fabricated scaffolds. The cell test demonstrated that the 3D-printed scaffolds are compatible with myoblast cells, which enabled the proliferation and attachment of cells, revealing a nontoxic behavior. The developed nanocellulose-based monolithic scaffolds have the advantages of allowing fast and inexpensive production, affording dimensional stability, drying, and rewetting (thus facilitating packaging, transport, and sterilization), and displaying high compatibility with cells.

\section{ASSOCIATED CONTENT}

\section{Supporting Information}

The Supporting Information is available free of charge on the ACS Publications website at DOI: 10.1021/acs.biomac.9b00527.

Atomic force microscopy amplitude error images; rheological behavior of nanocellulose inks (PDF)

3D printing with CNF inks and details of the TOCNF filaments (MP4) (MP4) (MP4)

\section{AUTHOR INFORMATION}

\section{Corresponding Authors}

*E-mail: helder.santos@helsinki.fi. Phone: +358 (0) 40846 1248 (H.A.S.).

*E-mail: orlando.rojas@aalto.fi. Phone: +358 (0)505124227 (O.J.R.).

ORCID ${ }^{\circ}$

Wenchao Xiang: 0000-0003-4281-3109

Rafael Grande: 0000-0001-7817-3698

Hélder A. Santos: 0000-0001-7850-6309

Orlando J. Rojas: 0000-0003-4036-4020

\section{Author Contributions}

The manuscript was written through contributions of all authors. All authors have given approval to the final version of the manuscript.

\section{Notes}

The authors declare no competing financial interest.

\section{ACKNOWLEDGMENTS}

We acknowledge funding support by the Academy of Finland's Biofuture 2025 program under project 2228357-4 (3D Manufacturing of Novel Biomaterials) and the European Research Council (ERC Advanced) under the European Union's Horizon 2020 research and innovation programme (grant agreement No 788489) and HiLIFE Research Funds and São Paulo Research Foundation (FAPESP) for the BEPE grant 2017/20891-8. This work made use of the facilities of Aalto University's Nanomicroscopy Center. In addition, the authors acknowledge the Electron Microscopy and Light Microscopy Units, Institute of Biotechnology, University of Helsinki, for providing laboratory facilities and assistance.

\section{REFERENCES}

(1) Markstedt, K.; Escalante, A.; Toriz, G.; Gatenholm, P. Biomimetic Inks Based on Cellulose Nano Fi Brils and CrossLinkable Xylans for 3D Printing. ACS Appl. Mater. Interfaces 2017, 9, 40878-40886.

(2) Sultan, S.; Siqueira, G.; Zimmermann, T.; Mathew, A. P. 3D Printing of Nano-Cellulosic Biomaterials for Medical Applications. Curr. Opin. Biomed. Eng. 2017, 2, 29-34.

(3) Rees, A.; Powell, L. C.; Chinga-Carrasco, G.; Gethin, D. T.; Syverud, K.; Hill, K. E.; Thomas, D. W. 3D Bioprinting of Carboxymethylated-Periodate Oxidized Nanocellulose Constructs for Wound Dressing Applications. BioMed Res. Int. 2015, 2015, No. 925757.

(4) Leppiniemi, J.; Lahtinen, P.; Paajanen, A.; Mahlberg, R.; MetsäKortelainen, S.; Pinomaa, T.; Pajari, H.; Vikholm-Lundin, I.; Pursula, P.; Hytönen, V. P. 3D-Printable Bioactivated Nanocellulose-Alginate Hydrogels. ACS Appl. Mater. Interfaces 2017, 9, 21959-21970. 
(5) Visscher, D. O.; Farré-Guasch, E.; Helder, M. N.; Gibbs, S.; Forouzanfar, T.; van Zuijlen, P. P.; Wolff, J. Advances in Bioprinting Technologies for Craniofacial Reconstruction. Trends Biotechnol. 2016, 34, 700-710.

(6) Rees, A.; Gethin, D.; Powell, L.; Chinga-Carrasco, G.; Claypole, T.; Deganello, D.; Hill, K. E.; Thomas, D. W.; Syverud, K. Studies on the 3D Printing of Nanocellulose Structures. In Advances in Printing and Media Technology, 2014; pp 91-95.

(7) Derakhshanfar, S.; Mbeleck, R.; Xu, K.; Zhang, X.; Zhong, W.; Xing, M. 3D Bioprinting for Biomedical Devices and Tissue Engineering: A Review of Recent Trends and Advances. Bioact. Mater. 2018, 3, 144-156.

(8) Martínez Ávila, H.; Schwarz, S.; Rotter, N.; Gatenholm, P. 3D Bioprinting of Human Chondrocyte-Laden Nanocellulose Hydrogels for Patient-Specific Auricular Cartilage Regeneration. Bioprinting 2016, 1-2, 22-35.

(9) Bishop, E. S.; Mostafa, S.; Pakvasa, M.; Luu, H. H.; Lee, M. J.; Wolf, J. M.; Ameer, G. A.; He, T. C.; Reid, R. R. 3-D Bioprinting Technologies in Tissue Engineering and Regenerative Medicine: Current and Future Trends. Genes Dis. 2017, 4, 185-195.

(10) Hua, K. A. I. Nanocellulose for Biomedical Applications. Doctoral Thesis, Uppsala University, 2015.

(11) Murphy, S. V.; Atala, A. Review 3D Bioprinting of Tissues and Organs. Nat. Biotechnol. 2014, 32, 773-785.

(12) Schaffner, M.; Rühs, P. A.; Coulter, F.; Kilcher, S.; Studart, A. R. 3D Printing of Bacteria into Functional Complex Materials. Sci. Adv. 2017, 3, No. eaao6804.

(13) Ventola, C. L. Medical Applications for 3D Printing: Current and Projected Uses. Pharm. Ther. 2014, 39, 704-711.

(14) Li, V. C. F.; Mulyadi, A.; Dunn, C. K.; Deng, Y.; Qi, H. J. Direct Ink Write 3D Printed Cellulose Nanofiber Aerogel Structures with Highly Deformable, Shape Recoverable, and Functionalizable Properties. ACS Sustainable Chem. Eng. 2018, 6, 2011-2022.

(15) Ngo, T. D.; Kashani, A.; Imbalzano, G.; Nguyen, K. T. Q.; Hui, D. Additive Manufacturing (3D Printing): A Review of Materials, Methods, Applications and Challenges. Composites, Part B 2018, 143, 172-196.

(16) Sultan, S.; Siqueira, G.; Zimmermann, T.; Mathew, A. P. 3D Printing of Nano-Cellulosic Biomaterials for Medical Applications. Curr. Opin. Biomed. Eng. 2017, 2, 29-34.

(17) Gu, B. K.; Choi, D. J.; Park, S. J.; Kim, M. S.; Kang, C. M.; Kim, C. H. 3-Dimensional Bioprinting for Tissue Engineering Applications. Biomater. Res. 2016, 20, 1-8.

(18) Bailey, C.; Stoyanov, S.; Tilford, T.; Tourloukis, G. In 3DPrinting and Electronic Packaging - Current Status and Future Challenges. 2016 IEEE 18th Electronics Packaging Technology Conference (EPTC), 2016; pp 1-4.

(19) Zeidler, H.; Klemm, D.; Böttger-Hiller, F.; Fritsch, S.; Le Guen, M. J.; Singamneni, S. 3D Printing of Biodegradable Parts Using Renewable Biobased Materials. Procedia Manuf. 2018, 21, 117-124.

(20) Sun, J.; Peng, Z.; Zhou, W.; Fuh, J. Y. H.; Hong, G. S.; Chiu, A. A Review on 3D Printing for Customized Food Fabrication. Procedia Manuf. 2015, 1, 308-319.

(21) Kidwell, J. Report: Best Practices and Applications of 3D Printing in the Construction Industry; San Luis Obispo: California, 2018.

(22) Zhu, L.; Li, N.; Childs, P. R. N. Light-Weighting in Aerospace Component and System Design. Propul. Power Res. 2018, 7, 103-119.

(23) Mertz, L. New World of 3-D Printing Offers "Completely New Ways of Thinking". IEEE Pulse 2013, 4, 12-14.

(24) Szost, B. A.; Terzi, S.; Martina, F.; Boisselier, D.; Prytuliak, A.; Pirling, T.; Hofmann, M.; Jarvis, D. J. A Comparative Study of Additive Manufacturing Techniques: Residual Stress and Microstructural Analysis of CLAD and WAAM Printed Ti-6Al-4V Components. Mater. Des. 2016, 89, 559-567.

(25) Park, M.; Lee, D.; Hyun, J. Nanocellulose-Alginate Hydrogel for Cell Encapsulation. Carbohydr. Polym. 2015, 116, 223-228.

(26) Piras, C. C.; Fernández-Prieto, S.; De Borggraeve, W. M. Nanocellulosic Materials as Bioinks for 3D Bioprinting. Biomater. Sci. 2017, 5, 1988-1992.
(27) Liu, J.; Cheng, F.; Grénman, H.; Spoljaric, S.; Seppälä, J.; Eriksson, J. E.; Willför, S.; Xu, C. Development of Nanocellulose Scaffolds with Tunable Structures to Support 3D Cell Culture. Carbohydr. Polym. 2016, 148, 259-271.

(28) Karim, Z.; Afrin, S. Cell \& Developmental Biology Nanocellulose as Novel Supportive Functional Material for Growth and Development of Cells. Cell Dev. Biol. 2015, 4, 2.

(29) Wang, J.; Chiappone, A.; Roppolo, I.; Shao, F.; Fantino, E.; Lorusso, M.; Rentsch, D.; Dietliker, K.; Pirri, C. F.; Grützmacher, H. All-in-One Cellulose Nanocrystals for 3D Printing of Nanocomposite Hydrogels. Angew. Chem., Int. Ed. 2018, 57, 2353-2356.

(30) Hauggaard, H.; Bergström Neda Keshavarzi, L. Report: 3D Printing of Zeolite-Cellulose Nanofibril Composites; Stockholm, Sweden, 2014.

(31) Sultan, S.; Abdelhamid, H. N.; Zou, X.; Mathew, A. P. CelloMOF: Nanocellulose Enabled 3D Printing of Metal-Organic Frameworks. Adv. Funct. Mater. 2018, 29, No. 1805372.

(32) Xu, W.; Wang, X.; Sandler, N.; Xu, C. Three-Dimensional Printing of Wood-Derived Biopolymers: A Review Focused on Biomedical Applications. ACS Sustainable Chem. Eng. 2018, 6, 56635680.

(33) Markstedt, K.; Mantas, A.; Tournier, I.; Martínez Ávila, H.; Hägg, D.; Gatenholm, P. 3D Bioprinting Human Chondrocytes with Nanocellulose-Alginate Bioink for Cartilage Tissue Engineering Applications. Biomacromolecules 2015, 16, 1489-1496.

(34) Laurén, P. Biomedical Applications of Nanofibrillar Cellulose. Doctoral Thesis, Helsinki University, 2018.

(35) Bhattacharya, M.; Malinen, M. M.; Lauren, P.; Lou, Y. R.; Kuisma, S. W.; Kanninen, L.; Lille, M.; Corlu, A.; Guguen-Guillouzo, C.; Ikkala, O.; Laukkanen, A.; Urtti, A.; Yliperttula, M. Nanofibrillar Cellulose Hydrogel Promotes Three-Dimensional Liver Cell Culture. J. Controlled Release 2012, 164, 291-298.

(36) Xu, C.; Molino, B. Z.; Wang, X.; Cheng, F.; Xu, W.; Molino, P.; Bacher, M.; Su, D.; Rosenau, T.; Willför, S.; Wallace, G. 3D Printing of Nanocellulose Hydrogel Scaffolds with Tunable Mechanical Strength towards Wound Healing Application. J. Mater. Chem. B 2018, 6, 7066-7075.

(37) Chakrabarty, A.; Teramoto, Y. Recent Advances in Nanocellulose Composites with Polymers: A Guide for Choosing Partners and How to Incorporate Them. Polymers 2018, 10, 1-47.

(38) Kärki, P. 3D Printing of Cellulose-Based Materials. Master Thesis, Aalto University, 2017.

(39) Narayanan Narasimhan, B. Chemically Programmed Timing for Nanocellulose Colloidal Gelation. Master Thesis, Aalto University, 2017.

(40) Markstedt, K.; Mantas, A.; Tournier, I.; Martínez Ávila, H.; Hägg, D.; Gatenholm, P. 3D Bioprinting Human Chondrocytes with Nanocellulose - Alginate Bioink for Cartilage Tissue Engineering Applications. Biomacromolecules 2015, 16, 1489-1496.

(41) Kovacs, T.; Naish, V.; Connor, B. O.; Blaise, C.; Gagné, F.; Hall, L.; Trudeau, V.; Martel, P. An Ecotoxicological Characterization of Nanocrystalline Cellulose (NCC). Nanotoxicology 2010, 4, 255270.

(42) Yang, G.; Xiao, Z.; Long, H.; Ma, K.; Zhang, J.; Ren, X.; Zhang, $\mathrm{J}$. Assessment of the Characteristics and Biocompatibility of Gelatin Sponge Scaffolds Prepared by Various Crosslinking Methods. Sci. Rep. 2018, 8, No. 1616

(43) Poursamar, S. A.; Lehner, A. N.; Azami, M.; Ebrahimi-Barough, S.; Samadikuchaksaraei, A.; Antunes, A. P. M. The Effects of Crosslinkers on Physical, Mechanical, and Cytotoxic Properties of Gelatin Sponge Prepared via in-Situ Gas Foaming Method as a Tissue Engineering Scaffold. Mater. Sci. Eng. C 2016, 63, 1-9.

(44) Jorfi, M.; Foster, E. J. Recent Advances in Nanocellulose for Biomedical Applications. J. Appl. Polym. Sci. 2015, 132, No. 41719.

(45) Bacakova, L.; Pajorova, J.; Bacakova, M.; Skogberg, A.; Kallio, P.; Kolarova, K.; Svorcik, V. Versatile Application of Nanocellulose: From Industry to Skin Tissue Engineering and Wound Healing. Nanomaterials 2019, 9, 164. 
(46) Shimotoyodome, A.; Suzuki, J.; Kumamoto, Y.; Hase, T.; Isogai, A. Regulation of Postprandial Blood Metabolic Variables by TEMPO-Oxidized Cellulose Nanofibers. Biomacromolecules 2011, 12, 3812-3818.

(47) Lin, N.; Dufresne, A. Nanocellulose in Biomedicine: Current Status and Future Prospect. Eur. Polym. J. 2014, 59, 302-325.

(48) Lang, N.; Merkel, E.; Fuchs, F.; Schumann, D.; Klemm, D.; Kramer, F.; Mayer-Wagner, S.; Schroeder, C.; Freudenthal, F.; Netz, H.; Kozlik-Feldmann, R.; Sigler, M. Bacterial Nanocellulose as a New Patch Material for Closure of Ventricular Septal Defects in a Pig Model. Eur. J. Cardio-thoracic Surg. 2015, 47, 1013-1021.

(49) Chen, P. H.; Liao, H. C.; Hsu, S. H.; Chen, R. S.; Wu, M. C.; Yang, Y. F.; Wu, C. C.; Chen, M. H.; Su, W. F. A Novel Polyurethane/Cellulose Fibrous Scaffold for Cardiac Tissue Engineering. RSC Adv. 2015, 5, 6932-6939.

(50) De France, K. J.; Yager, K. G.; Chan, K. J. W.; Corbett, B.; Cranston, E. D.; Hoare, T. Injectable Anisotropic Nanocomposite Hydrogels Direct in Situ Growth and Alignment of Myotubes. Nano Lett. 2017, 17, 6487-6495.

(51) Chang, C. W.; Wang, M. J. Preparation of Microfibrillated Cellulose Composites for Sustained Release of $\mathrm{H} 2 \mathrm{O} 2$ or $\mathrm{O} 2$ for Biomedical Applications. ACS Sustainable Chem. Eng. 2013, 1, 11291134.

(52) He, X.; Xiao, Q.; Lu, C.; Wang, Y.; Zhang, X.; Zhao, J.; Zhang, W.; Zhang, X.; Deng, Y. Uniaxially Aligned Electrospun All-Cellulose Nanocomposite Nanofibers Reinforced with Cellulose Nanocrystals: Scaffold for Tissue Engineering. Biomacromolecules 2014, 15, 618627.

(53) Favi, P. M.; Benson, R. S.; Neilsen, N. R.; Hammonds, R. L.; Bates, C. C.; Stephens, C. P.; Dhar, M. S. Cell Proliferation, Viability, and in Vitro Differentiation of Equine Mesenchymal Stem Cells Seeded on Bacterial Cellulose Hydrogel Scaffolds. Mater. Sci. Eng. C 2013, 33, 1935-1944.

(54) Guo, J.; Filpponen, I.; Su, P.; Laine, J.; Rojas, O. J. Attachment of Gold Nanoparticles on Cellulose Nanofibrils via Click Reactions and Electrostatic Interactions. Cellulose 2016, 23, 3065-3075.

(55) Tripathi, A.; Ago, M.; Khan, S. A.; Rojas, O. J. Heterogeneous Acetylation of Plant Fibers into Micro- and Nanocelluloses for the Synthesis of Highly Stretchable, Tough, and Water-Resistant CoContinuous Filaments via Wet-Spinning. ACS Appl. Mater. Interfaces 2018, 10, 44776-44786.

(56) Fujisawa, S.; Okita, Y.; Fukuzumi, H.; Saito, T.; Isogai, A. Preparation and Characterization of TEMPO-Oxidized Cellulose Nanofibril Films with Free Carboxyl Groups. Carbohydr. Polym. 2011, 84, 579-583.

(57) Saito, T.; Isogai, A. TEMPO-Mediated Oxidation of Native Cellulose. The Effect of Oxidation Conditions on Chemical and Crystal Structures of the Water-Insoluble Fractions. Biomacromolecules 2004, 1983-1989.

(58) London, E.; Brown, D. A. Insolubility of Lipids in Triton X100: Physical Origin and Relationship to Sphingolipid/Cholesterol Membrane Domains (Rafts). Biochim. Biophys. Acta 2000, 1508, 182195.

(59) Morais, J. P.; de Freitas Rosa, M.; Nascimento, L. D.; do Nascimento, D. M.; Cassales, A. R. Extraction and Characterization of Nanocellulose Structures from Raw Cotton Linter. Carbohydr. Polym. 2013, 91, 229-235.

(60) Isogai, A.; Saito, T.; Fukuzumi, H. TEMPO-Oxidized Cellulose Nanofiber. Nanoscale 2011, 3, 71-85.

(61) Moberg, T.; Sahlin, K.; Yao, K.; Geng, S.; Westman, G.; Zhou, Q.; Oksman, K.; Rigdahl, M. Rheological Properties of Nanocellulose Suspensions: Effects of Fibril/Particle Dimensions and Surface Characteristics. Cellulose 2017, 24, 2499-2510.

(62) Klemm, D.; Kramer, F.; Moritz, S.; Lindström, T.; Ankerfors, M.; Gray, D.; Dorris, A. Nanocelluloses: A New Family of NatureBased Materials. Angew. Chem., Int. Ed. 2011, 50, 5438-5466.

(63) Huan, S.; Ajdary, R.; Bai, L.; Klar, V.; Rojas, O. J. Low Solids Emulsion Gels Based on Nanocellulose for 3D-Printing. Biomacromolecules 2019, 20, 635-644.
(64) Habibi, Y. Key Advances in the Chemical Modification of Nanocelluloses. Chem. Soc. Rev. 2014, 43, 1519-1542.

(65) Lundahl, M. J.; Cunha, A. G.; Rojo, E.; Papageorgiou, A. C.; Rautkari, L.; Arboleda, J. C.; Rojas, O. J. Strength and Water Interactions of Cellulose i Filaments Wet-Spun from Cellulose Nanofibril Hydrogels. Sci. Rep. 2016, 6, No. 30695.

(66) Jiang, F.; Hsieh, Y. Lo Super Water Absorbing and Shape Memory Nanocellulose Aerogels from TEMPO-Oxidized Cellulose Nanofibrils via Cyclic Freezing-Thawing. J. Mater. Chem. A 2014, 2, $350-359$.

(67) Halib, N.; Perrone, F.; Id, M. C.; Dapas, B.; Farra, R.; Abrami, M.; Chiarappa, G.; Forte, G.; Zanconati, F.; Pozzato, G.; Murena, L.; Fiotti, N.; Liposin, R.; Cansolino, L.; Grassi, G.; Grassi, M. Potential Applications of Nanocellulose-Containing Materials in the Biomedical Field. Materials 2017, 10, No. 977.

(68) Aegerter, M. A.; Leventis, N.; Koebel, M. Aerogels Handbook; Springer: New York, 2011.

(69) Chang, H.-I.; Wang, Y. Cell Responses to Surface and Architecture of Tissue Engineering Scaffolds. In Regenerative Medicine and Tissue Engineering - Cells and Biomaterials; Eberli, D., Ed.; InTech: Taiwan, Australia, 2011; pp 569-588.

(70) Sencadas, V.; Ribeiro, S.; Gama, F. M.; Ribeiro, C.; Martins, P. M.; Lanceros-Méndez, S.; Gomes, A. C. Effect of Poling State and Morphology of Piezoelectric Poly(Vinylidene Fluoride) Membranes for Skeletal Muscle Tissue Engineering. RSC Adv. 2013, 3, No. 17938.

(71) Parizek, M.; Bacakova, L.; Filova, E.; Svorcik, V.; Ruml, T. Modulation of Cell Adhesion, Proliferation and Differentiation on Materials Designed for Body Implants. Biotechnol. Adv. 2011, 29, 739-767. 